\title{
Proposal of a New Risk Score for Patients Treated with Transarterial Chemoembolization due to Recurrent Hepatocellular Carcinoma after Curative Resection: A Multicenter Study
}

\author{
Mi Young Jeon ${ }^{1,2}$, Beom Kyung Kim ${ }^{1,2}$, Jun Yong Park ${ }^{1,2}$, Do Young Kim ${ }^{1,2}$, Sang Hoon Ahn ${ }^{1,2}$, Kwang-Hyub Han ${ }^{1,2}$, Jeong- \\ Hoon Lee ${ }^{3}$, Su Jong $\mathrm{Yu}^{3}$, Yoon Jun $\mathrm{Kim}^{3}$, Jung-Hwan Yoon ${ }^{3}$, Eun Ju Cho ${ }^{3}$, and Seung Up Kim ${ }^{1,2}$ \\ ${ }^{1}$ Department of Internal Medicine, Institute of Gastroenterology, Yonsei University College of Medicine, ${ }^{2}$ Yonsei Liver Center, Severance \\ Hospital, and ${ }^{3}$ Department of Internal Medicine and Liver Research Institute, Seoul National University College of Medicine, Seoul, Korea
}

Background/Aims: Prognostic models are lacking for patients with recurrent hepatocellular carcinoma (HCC) following surgical resection. This study devised and validated a new hepatoma arterial-embolization prognostic (HAP) score optimized for use in patients undergoing treatment with transarterial chemoembolization (TACE) for recurrence subsequent to surgical resection of HCC. Methods: Training cohort $(n=424)$ and validation cohort $(n=350)$ patients with recurrent HCC after resection treated with TACE between 2003 and 2016 were enrolled. Cox regression and area under the receiver operating characteristic curve (AUC) analyses were used to identify risk factors for survival and to calculate the predictive performance of risk scores, respectively. Results: The median age of the study population was 59.2 years. $\alpha$-Fetoprotein $>400 \mathrm{ng} / \mathrm{mL}$ (hazard ratio $[\mathrm{HR}]=1.815$ ), serum albumin $\leq 3.5 \mathrm{~g} / \mathrm{dL}(\mathrm{HR}=1.966)$, tumor number $\geq 2$ $(H R=1.425)$, tumor size $>5 \mathrm{~cm}$ at resection or recurrence $(H R=1.356)$, segmental portal vein invasion at resection or recurrence $(H R=2.032)$, and time from resection to recurrence $\leq 1$ years $(H R=1.849)$ independently predicted survival (all $p<0.05)$. The postoperative HAP ( $\mathrm{pHAP}$ ) model based on the rounded HRs of these variables showed an AUC of 0.723 for predicting survival at 3 years, which was significantly higher than AUCs of other HAP-based models, including HAP, modified HAP, and modified HAP-II scores (0.578-0.621) (all $p<0.05)$. The accuracy of pHAP was maintained in the entire cohort ( $n=774$; $A U C=0.776$ at 3 years). Conclusions: $A$ new pHAP score optimized for patients treated with TACE due to recurrent $\mathrm{HCC}$ after resection showed acceptable accuracy and was externally validated. Further studies of means by which to select treatment options other than TACE for highrisk patients according to pHAP scores are warranted. (Gut Liver 2020;14:477-485)

Key Words: Chemoembolization; Surgery; Recurrence; Carcinoma, hepatocellular

\section{INTRODUCTION}

Hepatocellular carcinoma (HCC) is a common cancer, and the third most common cause of cancer-associated death. ${ }^{1}$ With active surveillance programs supporting the detection of early stage HCC, the number of patients eligible for surgical treatment for HCC has increased..$^{1-3}$ Although improvements in surgical techniques ${ }^{3,4}$ and adequate selection criteria for resection have led to significant gains in survival and reductions in postoperative morbidity and mortality, the long-term prognosis remains unsatisfactory owing to the recurrence of HCC despite surgical resection. $^{5}$

Among the many treatment options for HCC that recurs following surgical resection, transarterial chemoembolization (TACE) has been shown to offer survival advantages in several randomized trials and a subsequent systematic review. ${ }^{6-9}$ Accordingly, TACE might be of use in treating recurrent HCC of Barcelona Clinic Liver Cancer (BCLC) intermediate stage B or early stage HCC not indicated for other curative treatments. ${ }^{10}$ However, it can be postulated that survival might vary greatly after TACE treatment for recurrent HCC due to variances in liver

Correspondence to: Seung Up Kim ${ }^{\mathrm{a}}$ and Eun Ju Cho ${ }^{\mathrm{b}}$

${ }^{a}$ Department of Internal Medicine, Yonsei University College of Medicine, 50 Yonsei-ro, Seodaemun-gu, Seoul 03722, Korea

Tel: +82-2-2228-1982, Fax: +82-2-393-6884, E-mail: ksukorea@yuhs.ac

${ }^{b}$ Department of Internal Medicine and Liver Research Institute, Seoul National University College of Medicine, 101 Daehak-ro, Jongno-gu, Seoul 03080, Korea

Tel: +82-2-2072-2242, Fax: +82-2-743-6701, E-mail: creatioex@gmail.com

Received on March 29, 2019. Revised on June 9, 2019. Accepted on June 27, 2019. Published online October $25,2019$. pISSN 1976-2283 eISSN 2005-1212 https://doi.org/10.5009/gnl19111

(c) This is an Open Access article distributed under the terms of the Creative Commons Attribution Non-Commercial License (http://creativecommons.org/licenses/by-nc/4.0) which permits unrestricted non-commercial use, distribution, and reproduction in any medium, provided the original work is properly cited. 
function and tumor burden upon HCC recurrence and the length of time till recurrence after surgical resection. ${ }^{11}$ Thus, it is of paramount importance to select candidates who would benefit most from TACE for recurrent HCC after surgical resection.

Recently, hepatoma arterial-embolization prognostic (HAP) score, which is composed of four variables, including $\alpha$-fetoprotein (AFP), tumor size, serum albumin, and total bilirubin, has been shown to predict outcomes following TACE. ${ }^{12}$ In addition, two adjusted versions of the HAP score have also been suggested: the modified HAP (mHAP) score, which excludes total bilirubin from the HAP score, ${ }^{13}$ and the mHAP-II score, which adds tumor number as one of the constituent variables. ${ }^{14}$ However, it is not known whether these risk scores show acceptable accuracy when applied to patients with recurrent HCC after surgical resection.

Thus, in this multicenter, retrospective study, we aimed to establish a new postoperative HAP (pHAP) score optimized for use in patients treated with TACE due to recurrent HCC following surgical resection and to validate the score externally. In addition, we compared the prognostic accuracy of the new model to the prognostic accuracy of existing HAP-related risk scores in the present study cohort.

\section{MATERIALS AND METHODS}

\section{Patient eligibility}

In this retrospective multicenter cohort study, we included consecutive patients undergoing treatment with TACE for recurrence subsequent to surgical resection of HCC between 2003 and 2015 ( $n=424$ for the training cohort, Severance Hospital, Yonsei University College of Medicine) and 2003 to 2016 ( $n=350$ for the validation cohort, Seoul National University Hospital, Seoul National University College of Medicine). The institutions in our study had extensive experience in the treatment of HCC using TACE.

Study exclusion criteria were (1) treatment modality other than TACE as first-line therapy; (2) inadequate target lesions on radiological assessment (non-arterial enhancement or largest lesion $<1 \mathrm{~cm}$ ); (3) presence of an additional primary malignancy in another organ; (4) presence of extrahepatic tumor lesions; (5) presence of tumor invasion to the main portal vein; (6) history of liver transplantation; (7) other serious medical comorbidities that might affect survival; (8) Child-Pugh class C; (8) BCLC stage D; and (9) a follow-up duration $<6$ months (Fig. 1).

The study protocol was in accordance with the Declaration of Helsinki guidelines and was approved by the Institutional Review Boards of Severance Hospital and Seoul National University Hospital. Due to retrospective nature of this study, the need for informed consent from the participants was waived.

\section{Diagnosis and staging of HCC}

HCC was diagnosed in accordance with the guidelines proposed by the Korea Liver Cancer Study Group. ${ }^{15}$ Typical HCC findings on dynamic computed tomography or magnetic resonance imaging were increased arterial enhancement and decreased enhancement. ${ }^{15,16}$ The BCLC staging system was applied for tumor staging, as previously described. ${ }^{17}$

\section{TACE procedure and follow-up}

Prior to the TACE procedure, angiography was utilized to assess vascular anatomy, patency, and tumor vascularity. ${ }^{14}$ Conventional TACE was performed using a selective infusion of a mixture of $5 \mathrm{~mL}$ of iodized oil contrast medium (Lipiodol ${ }^{\circledR}$; Guerbet LLC, Bloomington, IN, USA) and either $50 \mathrm{mg}$ of doxorubicin or cisplatin at $2 \mathrm{mg} / \mathrm{kg}$ body weight, followed by embolization using gelatin sponge particles (Cutanplast ${ }^{\circledR}$; Mascia Brunelli Spa, Milan, Italy). Radiologic responses to TACE were defined based on the modified Response Evaluation Criteria in

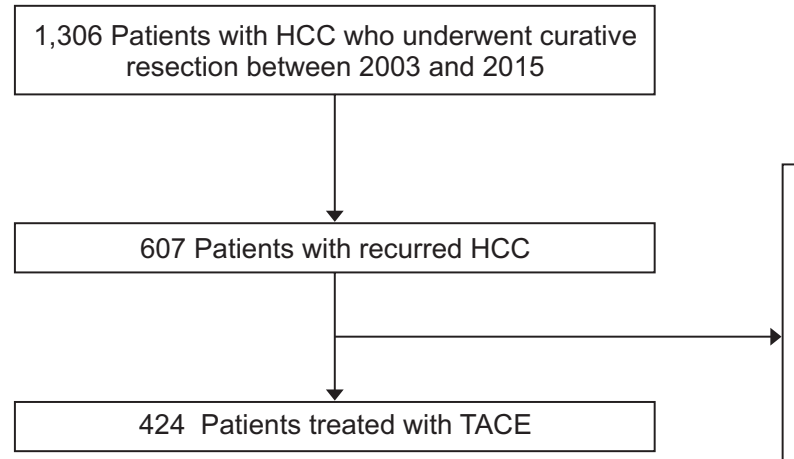

Other treatment modality except TACE

- Inadequate target lesions on radiologic assessment

- Presence of additional malignancies in another organ

- Presence of extrahepatic tumor lesions

- Presence of tumor invasion to the main portal vein

- Presence of liver transplantation history

- Other serious medical comorbidities that might affect the survival

- Child-Pugh classification C

- BCLC stage D

- Duration of follow-up $<6$ months

Fig. 1. Study population flowchart. A total of 1,306 patients with hepatocellular carcinoma (HCC) who underwent curative resection between 2003 and 2015 were placed in our training cohort. After the application of exclusion criteria, the training cohort consisted of 424 patients who underwent transarterial chemoembolization (TACE) due to recurrent HCC following curative resection (Yonsei University). Similarly, the validation cohort consisted of 350 patients with HCC who underwent curative resection between 2003 and 2016 and were treated with TACE due to recurrent HCC (Seoul National University).

BCLC, Barcelona Clinic Liver Cancer. 
Solid Tumours on computed tomography or magnetic resonance imaging. ${ }^{18}$ TACE was repeated at 6- to 8-week intervals on an "on-demand" until achievement of a complete response. ${ }^{18}$

\section{Study design}

This study was conducted in four parts. First, we attempted to identify variables associated with survival following TACE for recurrent HCC after surgical resection in the training cohort. Second, we estimated the accuracy of HAP-based risk models (Supplementary Table 1) to predict survival after TACE in the training set. Third, we established a new risk model optimized for patients in the study who experienced recurrence of HCC despite surgical resection and calculated the accuracy of the model, which was compared with the accuracy of other HAPrelated risk models. Lastly, the accuracy of the new risk model was validated in the validation set.

\section{Statistical analysis}

Patient and tumor characteristics at baseline are presented as a median (interquartile range) or number (\%) as appropriate. The Mann-Whitney test and Fisher exact test were used to compare characteristics between the study institutes, as appropriate. Survival and differences therein were analyzed using the KaplanMeier method and log-rank test.

The influence of variables on survival was evaluated using univariate and multivariate Cox regression analyses. The predictive performances of HAP-related risk scores and the newly established risk score at the time of TACE were assessed using area under the receiver operating characteristic curves (AUCs) to predict mortality at 1-, 3- and 5-year follow-up. The AUCs were compared using the DeLong test.

To compare the homogeneity and discriminatory ability of HAP-related risk scores and the newly established risk score, the likelihood ratio test and the linear trend test were utilized. In addition, the Akaike information criteria were calculated to demonstrate which of the risk scores was more explanatory and informative for risk assessment for survival among the existing HAP-related risk scores and the newly established one (smaller Akaike information criteria indicates preferred risk score).

All p-values $<0.05$ were considered indicative of statistical significance. The statistical analyses were conducted using SPSS 23.0 for Windows (IBM Corp., Armonk, NY, USA) and MedCalc Software version 12.7.2 (MedCalc Software bvba, Ostend, Belgium).

\section{RESULTS}

\section{Baseline characteristics}

A flowchart describing selection of the study population is shown in Fig. 1. Between 2003 and 2015, a total of 1,306 patients with HCC underwent surgical resection. Of these, 424 patients underwent TACE due to recurrent HCC after surgical resection and were include in the training cohort. Similarly, the validation cohort consisted of 350 patients with HCC who received surgical resection between 2003 and 2016 and TACE due to recurrent HCC.

Baseline data doe the training cohort at the time of TACE are shown in Table 1. The median age was 59.2 years (interquartile

Table 1. Baseline Characteristics of the Patients at the Time of TACE due to Recurrent HCC after Curative Resection (Training Set, $\mathrm{n}=424$ )

\begin{tabular}{|c|c|}
\hline Variable & Value \\
\hline \multicolumn{2}{|l|}{ Demographic variable } \\
\hline Age, yr & $59.2(52.2-66.6)$ \\
\hline Male sex & $359(84.7)$ \\
\hline \multicolumn{2}{|l|}{ Etiology } \\
\hline HBV & $345(81.4)$ \\
\hline $\mathrm{HCV}$ & $36(8.5)$ \\
\hline Others & $43(10.1)$ \\
\hline \multicolumn{2}{|l|}{ Child-Pugh class } \\
\hline A & 415 (97.9) \\
\hline $\mathrm{B}$ & $9(2.1)$ \\
\hline \multicolumn{2}{|l|}{ BCLC stage } \\
\hline 0 & $133(31.4)$ \\
\hline A & $163(38.4)$ \\
\hline B & $103(24.3)$ \\
\hline $\mathrm{C}$ & $25(5.9)$ \\
\hline \multicolumn{2}{|l|}{ Laboratory variables } \\
\hline$\alpha$-Fetoprotein, $\mathrm{ng} / \mathrm{mL}$ & $7.5(3.0-75.5)$ \\
\hline Total bilirubin, mg/dL & $0.8(0.6-1.0)$ \\
\hline Serum albumin, g/dL & $4.0(3.7-4.3)$ \\
\hline \multicolumn{2}{|l|}{ Tumor variables } \\
\hline \multicolumn{2}{|l|}{ Tumor size, cm } \\
\hline$\leq 7 />7$ & $414(97.6) / 10(2.4)$ \\
\hline$\leq 5 />5$ & $409(96.5) / 15(3.5)$ \\
\hline \multicolumn{2}{|l|}{ Tumor number } \\
\hline Unifocal & $229(54.0)$ \\
\hline Multifocal & $195(46.0)$ \\
\hline Segmental portal vein invasion & $25(5.9)$ \\
\hline \multicolumn{2}{|l|}{ Tumor variables at the time of resection } \\
\hline \multicolumn{2}{|l|}{ Tumor size, cm } \\
\hline$\leq 7 />7$ & $381(89.9) / 43(10.1)$ \\
\hline$\leq 5 />5$ & $313(73.8) / 111(26.2)$ \\
\hline \multicolumn{2}{|l|}{ Tumor number } \\
\hline Unifocal & $354(83.5)$ \\
\hline Multifocal & $70(16.5)$ \\
\hline Segmental portal vein invasion & $32(7.5)$ \\
\hline Time from resection to recurrence, mo & $17.7(7.2-36.6)$ \\
\hline
\end{tabular}

Data are presented as the median (interquartile range) or number (\%). TACE, transarterial chemoembolization; HCC, hepatocellular carcinoma; HBV, hepatitis B virus; HCV, hepatitis C virus; BCLC, Barcelona Clinic Liver Cancer. 
range, 52.2-66.6 years) and 359 patients (84.7\%) were men. The most common etiology of HCC was hepatitis B virus (HBV) infection ( $\mathrm{n}=345,81.4 \%)$. A total of 415 patients (97.9\%) had well-preserved liver function (Child-Pugh class A), and 296 patients (69.8\%) were BCLC stage 0-A. Data for the validation cohort are shown in Supplementary Table 2. The comparisons among patients according to period of recurrence of HCC after resection ( $\leq 1$ or $>1$ year) in the training cohort were described in Supplementary Table 3. Age, BCLC stage, AFP, serum albumin, tumor size $>7 \mathrm{~cm}$, tumor number at the time of recurrence and tumor size $>5 \mathrm{~cm}$ at the time of resection demonstrated significant differences between patients with recurrences within and more than 1 -year of surgical resection (all $\mathrm{p}<0.05$ ).

\section{Survival after TACE due to recurred HCC and its risk fac- tors}

The survival rates at 1,3 , and 5 years after TACE, respectively, were $87.4 \%, 62.6 \%$, and $47.8 \%$, and the median survival was 57.1 months (interquartile range, 41.4-72.8 months) in the training cohort.

Table 2. Univariate Cox Regression Analysis to Identify Potential Risk Factors for Overall Mortality at the Time of TACE after Curative Resection (Training Set, $n=424$ )

\begin{tabular}{|c|c|c|}
\hline Variable & HR $(95 \%$ CI) & p-value \\
\hline Age & $0.991(0.979-1.003)$ & 0.123 \\
\hline Male ( $n=359)$ (vs female, $n=65$ ) & $1.105(0.778-1.570)$ & 0.577 \\
\hline Viral etiology ( $\mathrm{n}=381$ ) (vs non-viral, $\mathrm{n}=43$ ) & $1.128(0.733-1.735)$ & 0.584 \\
\hline \multicolumn{3}{|l|}{$\alpha$-Fetoprotein, $\mathrm{ng} / \mathrm{mL}$} \\
\hline$>400(\mathrm{n}=44)(\mathrm{vs} \leq 400, \mathrm{n}=380)$ & $2.050(1.425-2.949)$ & $<0.001$ \\
\hline \multicolumn{3}{|l|}{ Total bilirubin, mg/dL } \\
\hline$>1.7(\mathrm{n}=10)(\mathrm{vs} \leq 1.7, \mathrm{n}=414)$ & $1.054(0.468-2.371)$ & 0.899 \\
\hline \multicolumn{3}{|l|}{ Serum albumin, $\mathrm{g} / \mathrm{dL}$} \\
\hline$\leq 3.5(\mathrm{n}=57)(\mathrm{vs}>3.5, \mathrm{n}=367)$ & $2.012(1.445-2.801)$ & $<0.001$ \\
\hline \multicolumn{3}{|l|}{ Tumor variable } \\
\hline \multicolumn{3}{|l|}{ Tumor size, cm } \\
\hline$>5(\mathrm{n}=15)(\mathrm{vs} \leq 5, \mathrm{n}=409)$ & $2.459(1.400-4.320)$ & 0.002 \\
\hline Multifocal ( $\mathrm{n}=70$ ) (vs unifocal, $\mathrm{n}=354$ ) & $1.574(1.220-2.030)$ & $<0.001$ \\
\hline Segmental portal vein invasion $(n=25)$ & $2.925(1.820-4.702)$ & $<0.001$ \\
\hline \multicolumn{3}{|l|}{ Tumor variables at the time of resection } \\
\hline \multicolumn{3}{|l|}{ Tumor size, cm } \\
\hline$>5(\mathrm{n}=111)(\mathrm{vs} \leq 5, \mathrm{n}=313)$ & $1.345(1.018-1.775)$ & 0.037 \\
\hline Multifocal ( $\mathrm{n}=70$ ) (vs unifocal, $\mathrm{n}=354$ ) & $1.099(0.779-1.547)$ & 0.592 \\
\hline Segmental portal vein invasion $(n=32)$ & $1.845(1.208-2.817)$ & 0.005 \\
\hline \multicolumn{3}{|l|}{ Time from resection to recurrence, $\mathrm{yr}$} \\
\hline$\leq 1(\mathrm{n}=159)(\mathrm{vs}>1, \mathrm{n}=265)$ & $2.361(1.829-3.047)$ & $<0.001$ \\
\hline
\end{tabular}

TACE, transarterial chemoembolization; HR, hazard ratio; CI, confidence interval.

Table 3. Multivariate Cox Regression Analysis to Identify Independent Risk Factors for Poor Survival and the Corresponding Rounded Risk Score Based on pHAP Score (Training Set, $n=424$ )

\begin{tabular}{|c|c|c|c|}
\hline \multirow{2}{*}{ Variable } & \multicolumn{2}{|c|}{ Multivariate } & \multirow{2}{*}{$\begin{array}{l}\text { Allocation of rounded } \\
\text { score for pHAP score }\end{array}$} \\
\hline & HR (95\% CI) & p-value & \\
\hline$\alpha$-Fetoprotein (>400 ng/mL) & $1.815(1.242-2.651)$ & 0.002 & 1 \\
\hline Serum albumin $(\leq 3.5 \mathrm{~g} / \mathrm{dL})$ & $1.966(1.401-2.760)$ & $<0.001$ & 1 \\
\hline Tumor number at the time of recurrence $(\geq 2)$ & $1.425(1.093-1.859)$ & $<0.001$ & 1 \\
\hline Tumor size $(>5 \mathrm{~cm})$ at the time of resection or recurrence & $1.356(1.028-1.789)$ & 0.031 & 1 \\
\hline Segmental PVI at the time of resection or recurrence & $2.032(1.436-2.875)$ & $<0.001$ & 1 \\
\hline Time from resection to recurrence $(\leq 1 \mathrm{yr})$ & $1.849(1.412-2.422)$ & $<0.001$ & 1 \\
\hline
\end{tabular}

Risk group are classified into pHAP A (0-1 point), pHAP B ( 2 points), pHAP C ( 3 points), or pHAP D ( $\geq 4$ points), respectively.

pHAP, postoperative hepatoma arterial-embolization prognostic; HR, hazard ratio; CI, confidence interval; PVI, portal vein invasion. 
On univariate analyses, AFP level $>400 \mathrm{ng} / \mathrm{mL}$, serum albumin level $\leq 3.5 \mathrm{~g} / \mathrm{dL}$, multifocal tumor at the time of recurrence, tumor size $>5 \mathrm{~cm}$ and segmental portal vein invasion at the time of resection or recurrence, and time from resection to recurrence were risk factors for poor survival (all p<0.05) (Table 2). Subsequent multivariate analysis identified AFP level $>400 \mathrm{ng} /$ $\mathrm{mL}$, serum albumin level $\leq 3.5 \mathrm{~g} / \mathrm{dL}$, multiple tumors at the time of recurrence, tumor size $>5 \mathrm{~cm}$ at the time of resection or recurrence, segmental portal vein invasion at the time of resection or recurrence, and time from resection to recurrence $\leq 1$ years as independent risk factors for poor survival (Table 3).

Using these variables, we developed our pHAP model for patients treated with TACE due to recurrent HCC after surgical resection was developed and validated (Supplementary Tables 4 and 5). The pHAP score was defined as the sum of the points of the six variables, and patients were classified into pHAP A (0-1 point), pHAP B ( 2 points), pHAP C ( 3 points), or pHAP D ( $\geq 4$ points), respectively (Table 3 ).

\section{Predictive performance of pHAP and other HAP-related risk scores}

The AUC values of pHAP and other HAP-related risk scores were calculated and compared (Table 4). The AUC values of pHAP score were 0.799 at 1 year, 0.723 at 3 years, and 0.697 at 5 years (all $\mathrm{p}<0.001$ ). Comparison of the AUC values, revealed a significantly higher AUC for pHAP score than for the other HAP-related risk scores from 1 to 5 years (AUC: 0.697-0.799 for pHAP vs 0.565-0.682 for HAP, 0.567-0.681 for mHAP, and $0.618-0.728$ for mHAP-II) (all $\mathrm{p}<0.05$ ). Similar AUC values were identified in the entire cohort including the training and validation cohorts $(0.812$ at 1 year, 0.776 at 3 years, and 0.768 at 5 years) (Supplementary Table 6).

\section{Survival outcomes according to pHAP and other HAP- related risk scores}

Median survival and survival rates at 1 to 5 years in the training cohort are presented in Supplementary Table 7 according to pHAP and other HAP-related risk scores. Kaplan-Meier survival curves for each of the risk groups in the pHAP score model are depicted in Fig. 2. The survival curves of four risk groups based on pHAP score were significantly different (overall log-rank, $\mathrm{p}<0.001)$. Similar to the results observed in the training cohort, the survival curves of the four risk groups based on pHAP and the other HAP-related risk scores in the validation cohort and the entire cohort were significantly different (overall all $\mathrm{p}<0.001$ by log-rank test) (Supplementary Fig. 1).

\section{Prognostic accuracy of pHAP and other HAP-related risk scores}

Among the risk scores, pHAP score showed the highest homogeneity compared to the other HAP-related risk scores (likelihood ratio, 87.501 vs 32.207 to 46.437), the highest discriminatory ability (linear trend, 81.932 vs 30.977 to 45.583 ), and the lowest Akaike information criteria value $(2,536.533$ vs $2,560.487$ to $2,576.985)$, indicating the best prognostic performance in patients treated with TACE due to recurrent HCC after surgical resection (Table 5). When these were applied to the entire cohort, similar results were observed (Supplementary Table 8).

\section{DISCUSSION}

Recent randomized controlled trials and meta-analyses have

Table 4. Predictive Performance of HAP, mHAP-I, mHAP-II, and pHAP ( $n=424$ in Training, $n=350$ in Validation)

\begin{tabular}{|c|c|c|c|c|c|c|c|}
\hline \multirow{3}{*}{ Follow-up } & \multicolumn{7}{|c|}{ Training cohort } \\
\hline & \multicolumn{2}{|c|}{ HAP } & \multicolumn{2}{|c|}{ mHAP } & \multicolumn{2}{|c|}{ mHAP-II } & \multirow{2}{*}{$\frac{\text { pHAP }}{\text { AUC (95\% CI) }}$} \\
\hline & AUC (95\% CI) & p-value ${ }^{\ddagger}$ & AUC (95\% CI) & p-value ${ }^{\ddagger}$ & AUC (95\% CI) & p-value ${ }^{\ddagger}$ & \\
\hline \multicolumn{8}{|l|}{ Entire } \\
\hline \multirow[t]{2}{*}{1 Year } & 0.682 & $<0.001$ & 0.681 & $<0.001$ & 0.728 & 0.004 & 0.799 \\
\hline & $(0.602-0.761)^{*}$ & & $(0.600-0.762)^{*}$ & & $(0.658-0.797)^{*}$ & & $(0.743-0.855)^{*}$ \\
\hline \multirow[t]{2}{*}{2 Years } & 0.617 & $<0.001$ & 0.616 & $<0.001$ & 0.669 & $<0.001$ & 0.743 \\
\hline & $(0.554-0.679)^{*}$ & & $(0.553-0.679)^{*}$ & & $(0.611-0.726)^{*}$ & & $(0.690-0.796)^{*}$ \\
\hline \multirow[t]{2}{*}{3 Years } & 0.578 & $<0.001$ & 0.580 & $<0.001$ & 0.621 & $<0.001$ & 0.723 \\
\hline & $(0.522-0.634)^{\dagger}$ & & $(0.524-0.636)^{\dagger}$ & & $(0.566-0.675)^{*}$ & & $(0.674-0.772)^{*}$ \\
\hline \multirow[t]{2}{*}{4 Years } & 0.579 & $<0.001$ & 0.581 & $<0.001$ & 0.627 & $<0.001$ & 0.713 \\
\hline & $(0.524-0.633)^{\dagger}$ & & $(0.527-0.636)^{\dagger}$ & & $(0.574-0.680)^{*}$ & & $(0.655-0.762)^{*}$ \\
\hline \multirow[t]{2}{*}{5 Years } & 0.565 & $<0.001$ & 0.567 & $<0.001$ & 0.618 & $<0.001$ & 0.697 \\
\hline & $(0.510-0.619)^{\dagger}$ & & $(0.513-0.622)^{\dagger}$ & & $(0.565-0.671)^{*}$ & & $(0.648-0.747)^{*}$ \\
\hline
\end{tabular}

HAP, hepatoma arterial-embolization prognostic; mHAP, modified HAP; pHAP, postoperative HAP; AUC, area under the receiver operating characteristic curve; $\mathrm{CI}$, confidence interval.

${ }^{*} \mathrm{p}<0.001 ;{ }^{\dagger} \mathrm{p}<0.05 ;{ }^{\dagger} \mathrm{p}$-value indicates a significant AUC for pHAP in the training set by the DeLong test. 

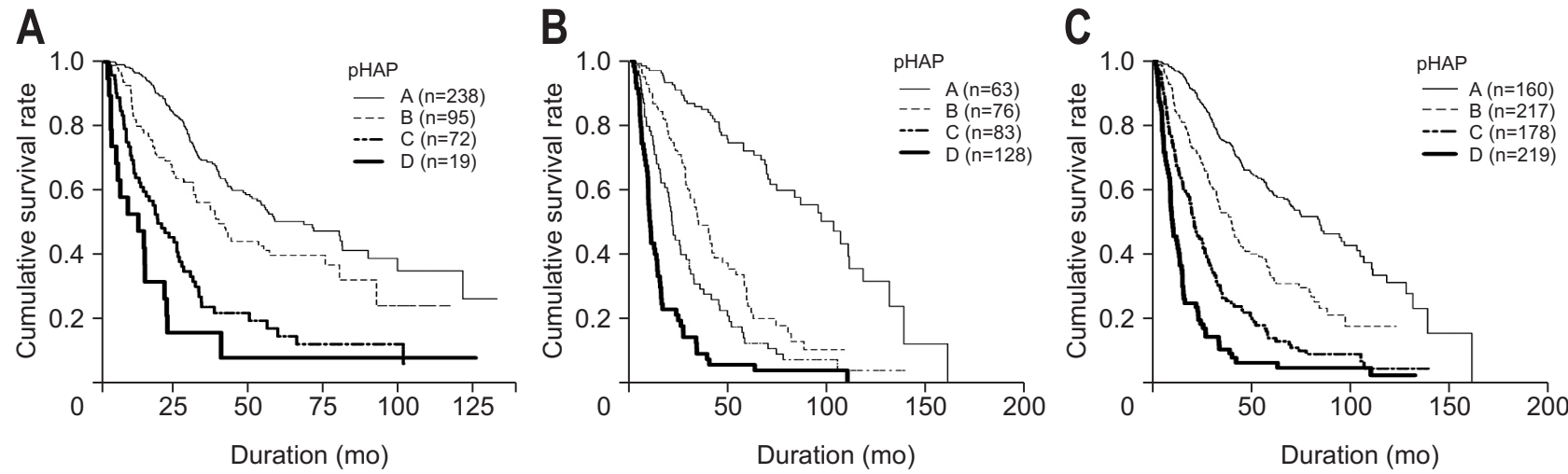

Fig. 2. Cumulative overall survival rate according to pHAP stratification (Kaplan-Meier curves). (A) Cumulative survival curves in the training set (overall, $p<0.001$; A vs B class, $p=0.007$; A vs $C$ class, $p<0.001$; A vs D class, $p<0.001$; all by log-rank test), (B) the validation set (overall, $p<0.001$; A vs $B$ class, $p<0.001$; A vs $C$ class, $p<0.001$; A vs D class, $p<0.001$; all by log-rank test), and (C) the entire cohort (overall, $p<0.001$; A vs B class, $\mathrm{p}<0.001$; B vs $\mathrm{C}$ class, $\mathrm{p}<0.001$; A vs $\mathrm{D}$ class, $\mathrm{p}<0.001$; all by log-rank test).

pHAP, postoperative hepatoma arterial-embolization prognostic.

Table 5. Prognostic Accuracy of Risk Scores during TACE to Predict Mortality in the Training Set

\begin{tabular}{lccc}
\hline Risk score & $\begin{array}{c}\text { Likelihood ratio } \\
\left(\chi^{2}\right)\end{array}$ & $\begin{array}{c}\text { Linear trend } \\
\left(\chi^{2}\right)\end{array}$ & AIC \\
\hline HAP & 32.207 & 30.977 & $2,576.985$ \\
mHAP & 41.077 & 39.273 & $2,567.679$ \\
mHAP-II & 46.437 & 45.583 & $2,560.487$ \\
pHAP & 87.501 & 81.932 & $2,536.533$ \\
\hline
\end{tabular}

The model with a higher $\chi^{2}$ value by the likelihood ratio and linear trend tests was considered the better model for homogeneity and discriminatory ability. Furthermore, lower values for the Akaike information criteria (AIC) were considered to indicate better discriminatory ability.

TACE, transarterial chemoembolization; HAP, hepatoma arterialembolization prognostic; mHAP, modified HAP; pHAP, postoperative HAP.

proven that TACE can provide survival benefit in patients with unresectable HCC. ${ }^{19}$ However, due to heterogeneity in liver function and tumor characteristics at the time of TACE, as well as individual differences regarding the performance of TACE among institutions, ${ }^{20}$ a wide-range of treatment outcomes has been reported. ${ }^{21}$ Thus, it has been important to distinguish patients who would benefit from TACE from those who would not and should hence receive alternative treatments, such as sorafenib or other palliative treatments. ${ }^{22,23}$

Although several previous studies have compared the ability of various staging systems to predict the survival in patients with unresectable HCC treated with TACE, ${ }^{24,25}$ several simple-touse risk stratification models, such as the HAP model ${ }^{12}$ and its modifications, ${ }^{13,14,22}$ have also been proposed. However, because no risk stratification model for TACE due to recurrent HCC after surgical resection has been available, our study aimed to identify independent risk factors for poor survival in this clinical setting and to develop and validate a new risk model. In this study, our new risk model, pHAP, demonstrated an AUC to pre- dict survival at 3 years of 0.723 , significantly higher than AUCs of other HAP-based models (AUC, 0.578 to 0.621) (all $\mathrm{p}<0.05$ between AUCs).

Our study has several strengths. First is the large sample size ( $n=774$ ) and the long-term follow-up period (median 57.1, up to 155.9 months). This gave us greater statistical power with which to identify prognostic factors for poor long-term prognosis and to establish a score model optimized for patients who undergoing TACE due to recurrent HCC after surgical resection. To the best of our knowledge, this study is the first to identify such prognostic factors, to establish a new predictive model (pHAP model), to achieve external validation thereof, and to demonstrate the superior predictive accuracy of the model with high homogeneity and discriminatory ability, compared to those of HAP and its modifications, in this clinical setting. Moreover, the pHAP model-maintained simplicity in score calculation, a feature that might be helpful for clinicians in use of the model in clinical practice.

Second, as the intense surveillance strategy with computed tomography or magnetic resonance imaging to detect recurrence is significantly different from the ultrasound-based surveillance for CHB-patients without HCC, a different risk stratification system is strongly required for patients who underwent TACE due to recurrent HCC after resection. Re-resection is generally infeasible in patients who have already undergone surgery due to technical difficulties, although these patients might have wellpreserved liver function with early stage recurrent HCC as a result of intensive follow-up. In comparison to previous studies of HAP and its modifications, ${ }^{12-14}$ the proportion of single tumors (54.0\% vs 37\% to $42 \%$ ) and small diameter of tumor size ( $\leq 7$ cm) $(97.6 \%$ vs $70 \%$ to $86 \%)$ were higher and the median AFP level was lower (7.7\% vs $15 \%$ to $28 \%$ ) in patients treated with TACE after resection. For these reasons, despite post-resection status, the median overall survival after the initial TACE in our cohort was significantly longer at 43.3 months, compared to 
13.7-36.2 months in previous studies in which HAP and its modifications were derived. ${ }^{12,13}$ This difference is the main reason why we tried to focus on the patients who underwent TACE due to recurrent HCC after surgical resection. However, because our cohort included patients treated with TACE after resection despite very early and early HCC stage, the results should be interpreted with caution.

Third, in contrast to previous studies proposing HAP models, ${ }^{12-14}$ total bilirubin level lacked predictive ability for survival. This finding can be partly explained based on the selection of our study population cohort. Because our cohort with recurrent HCC consisted of only patients who could endure previous surgical resection, well-preserved liver function might be maintained at the time of TACE due to recurrent HCC. Indeed, the proportion of patients with Child-Pugh class A liver function in our cohort was higher than those of previous studies (97.9\% vs $71 \%$ to $78 \%$ ). ${ }^{12,13}$ In addition, segmental portal vein invasion was selected as one of the independent predictors of poor prognosis. In Asian countries, segmental portal vein invasion has not been considered a contraindication for TACE. ${ }^{26}$ Indeed, the proportion of segmental portal vein invasion at TACE has not been reported negligible in previous studies. ${ }^{12,14}$ Thus, incorporation of segmental portal vein invasion into the pHAP model might be generally applicable. In addition, a shorter time interval from resection to TACE ( $<1$ year) was an independent poor prognostic factor. The selection of this variable can be easily supported by considering that the early recurrence of HCC after resection has been reported to be a prognostic factor for survival despite treatment, ${ }^{27}$ which in turn supports our rationale to develop a risk prediction model for recurrent HCC after resection that includes the time interval between TACE and resection as one of the constituent variables.

Our study also has several limitations. First, our study population consisted of Korean patients only, the majority of whom had HBV as the etiology for HCC (around 80\%). Therefore, validation of our findings in other ethnic populations with varying etiologies for HCC is strongly required. In addition, we retrospectively selected only patients who underwent TACE due to recurrence. However, the decision of whether to perform TACE or not can be significantly influenced by previous treatment modality, tumor number, and location and size of tumor, creating a possible selection bias. We attempted to overcome this potential bias by validating the pHAP model in a large-sized external cohort $(n=350)$ and demonstrating the applicability of the pHAP model in patients undergoing TACE after resection. Second, the overall diagnostic accuracy is not that high in contrast to those observed in previous studies of HAP and its modifications. Although the exact reason for these findings is not clear, this phenomenon can be explained in part by the selection of variables at the time of recurrence. Furthermore, potent antiviral therapy using nucleotide analogues for hepatitis $\mathrm{B}$ and direct-acting antivirals for hepatitis $\mathrm{C}$ might also have an impact on the overall survival of our cohort; a possibility that should be further investigated in future studies. Third, interinstitutional variability regarding technical issue and subjective decisions regarding treatment might have influenced our fina results. However, we attempted to validate the pHAP model in an external cohort and observed similar diagnostic accuracy between the training and validation cohorts. Lastly, although re-treatment strategies for TACE have been proposed, such as sequential use of various risk models of HAP and its modifications, ART (Assessment for Retreatment with TACE) score, SNACOR (tumor size and number, baseline AFP, Child-Pugh and objective radiological response), and $\mathrm{ABCR}$ score, ${ }^{28-30}$ the present study focused on selection of the optimal candidates for starting TACE as the first-line anticancer therapy for recurrent HCC.

In conclusion, we developed and externally validated a new HAP model (pHAP) with greater accuracy in patients treated with TACE due to the recurrent HCC following surgical resection. Further studies should investigate appropriate methods for selection of treatment options other than TACE for patients considered to be at high-risk according to pHAP score.

\section{CONFLICTS OF INTEREST}

No potential conflict of interest relevant to this article was reported.

\section{ACKNOWLEDGEMENTS}

This study was supported by the Basic Science Research Program through the National Research Foundation of Korea funded by the Ministry of Science, ICT \& Future Planning (grant number: 2016R1A1A1A05005138).

\section{AUTHOR CONTRIBUTIONS}

Study concept and design: E.J.C., S.U.K. Data acquisition: B.K.K., J.Y.P., D.Y.K., S.H.A., K.H.H., J.H.L., S.J.Y., Y.J.K., J.H.Y., E.J.C., S.U.K. Data analysis and interpretation: M.Y.J., E.J.C., S.U.K. Drafting of the manuscript; critical revision of the manuscript for important intellectual content: M.Y.J., E.J.C., S.U.K Statistical analysis: M.Y.J., E.J.C., S.U.K. Obtained funding: S.U.K Administrative, technical, or material support; study supervision: E.J.C., S.U.K

\section{ORCID}

Mi Young Jeon

Beom Kyung Kim

Jun Yong Park

Do Young Kim

Sang Hoon Ahn

Kwang-Hyub Han https://orcid.org/0000-0002-3980-4503 https://orcid.org/0000-0002-5363-2496 https://orcid.org/0000-0001-6324-2224 https://orcid.org/0000-0002-8327-3439 https://orcid.org/0000-0002-3629-4624 https://orcid.org/0000-0003-3960-6539 
Jeong-Hoon Lee

Su Jong Yu

Yoon Jun Kim

Jung-Hwan Yoon

Eun Ju Cho

Seung Up Kim

\section{REFERENCES}

1. El-Serag HB. Epidemiology of viral hepatitis and hepatocellular carcinoma. Gastroenterology 2012;142:1264-1273.

2. Yuen MF, Cheng CC, Lauder IJ, Lam SK, Ooi CG, Lai CL. Early detection of hepatocellular carcinoma increases the chance of treatment: Hong Kong experience. Hepatology 2000;31:330-335.

3. Lee JM, Jang BK, Lee YJ, et al. Survival outcomes of hepatic resection compared with transarterial chemoembolization or sorafenib for hepatocellular carcinoma with portal vein tumor thrombosis. Clin Mol Hepatol 2016;22:160-167.

4. Kim JY, Sinn DH, Gwak GY, et al. Transarterial chemoembolization versus resection for intermediate-stage (BCLC B) hepatocellular carcinoma. Clin Mol Hepatol 2016;22:250-258.

5. Tung-Ping Poon R, Fan ST, Wong J. Risk factors, prevention, and management of postoperative recurrence after resection of hepatocellular carcinoma. Ann Surg 2000;232:10-24.

6. Kim BK, Kim SU, Kim KA, et al. Complete response at first chemoembolization is still the most robust predictor for favorable outcome in hepatocellular carcinoma. J Hepatol 2015;62:1304-1310.

7. Park MS, Kim SU, Park JY, et al. Combination treatment of localized concurrent chemoradiation therapy and transarterial chemoembolization in locally advanced hepatocellular carcinoma with intrahepatic metastasis. Cancer Chemother Pharmacol 2013;71:165-173.

8. Llovet JM, Real MI, Montana X, et al. Arterial embolisation or chemoembolisation versus symptomatic treatment in patients with unresectable hepatocellular carcinoma: a randomised controlled trial. Lancet 2002;359:1734-1739.

9. Lo CM, Ngan H, Tso WK, et al. Randomized controlled trial of transarterial lipiodol chemoembolization for unresectable hepatocellular carcinoma. Hepatology 2002;35:1164-1171.

10. Bruix J, Sherman M; American Association for the Study of Liver Diseases. Management of hepatocellular carcinoma: an update. Hepatology 2011;53:1020-1022.

11. Jung KS, Kim JH, Kim SU, et al. Liver stiffness value-based risk estimation of late recurrence after curative resection of hepatocellular carcinoma: development and validation of a predictive model. PLoS One 2014;9:e99167.

12. Kadalayil L, Benini R, Pallan L, et al. A simple prognostic scoring system for patients receiving transarterial embolisation for hepatocellular cancer. Ann Oncol 2013;24:2565-2570.

13. Pinato DJ, Arizumi T, Allara E, et al. Validation of the hepatoma arterial embolization prognostic score in European and Asian populations and proposed modification. Clin Gastroenterol Hepa- tol 2015;13:1204-1208.

14. Park Y, Kim SU, Kim BK, et al. Addition of tumor multiplicity improves the prognostic performance of the hepatoma arterialembolization prognostic score. Liver Int 2016;36:100-107.

15. Korean Liver Cancer Study Group and National Cancer Center, Korea. Practice guidelines for management of hepatocellular carcinoma 2009. Korean J Hepatol 2009;15:391-423.

16. European Association for the Study of the Liver; European Organisation for Research and Treatment of Cancer. EASL-EORTC clinical practice guidelines: management of hepatocellular carcinoma. J Hepatol 2012;56:908-943.

17. Forner A, Reig ME, de Lope CR, Bruix J. Current strategy for staging and treatment: the BCLC update and future prospects. Semin Liver Dis 2010;30:61-74.

18. Kim BK, Kim KA, Park JY, et al. Prospective comparison of prognostic values of modified Response Evaluation Criteria in Solid Tumours with European Association for the Study of the Liver criteria in hepatocellular carcinoma following chemoembolisation. Eur J Cancer 2013;49:826-834.

19. Forner A, Gilabert M, Bruix J, Raoul JL. Treatment of intermediatestage hepatocellular carcinoma. Nat Rev Clin Oncol 2014;11:525-535.

20. Marelli L, Stigliano R, Triantos C, et al. Transarterial therapy for hepatocellular carcinoma: which technique is more effective? A systematic review of cohort and randomized studies. Cardiovasc Intervent Radiol 2007;30:6-25.

21. Raoul JL, Sangro B, Forner A, et al. Evolving strategies for the management of intermediate-stage hepatocellular carcinoma: available evidence and expert opinion on the use of transarterial chemoembolization. Cancer Treat Rev 2011;37:212-220.

22. Pinato DJ, Arizumi T, Jang JW, et al. Combined sequential use of HAP and ART scores to predict survival outcome and treatment failure following chemoembolization in hepatocellular carcinoma: a multi-center comparative study. Oncotarget 2016;7:4470544718.

23. Zhao Y, Cai G, Zhou L, et al. Transarterial chemoembolization in hepatocellular carcinoma with vascular invasion or extrahepatic metastasis: a systematic review. Asia Pac J Clin Oncol 2013;9:357364.

24. Takayasu K, Arii S, Ikai I, et al. Prospective cohort study of transarterial chemoembolization for unresectable hepatocellular carcinoma in 8510 patients. Gastroenterology 2006;131:461-469.

25. Georgiades CS, Liapi E, Frangakis C, et al. Prognostic accuracy of 12 liver staging systems in patients with unresectable hepatocellular carcinoma treated with transarterial chemoembolization. J Vasc Interv Radiol 2006;17:1619-1624.

26. Luo J, Guo RP, Lai EC, et al. Transarterial chemoembolization for unresectable hepatocellular carcinoma with portal vein tumor thrombosis: a prospective comparative study. Ann Surg Oncol 2011;18:413-420.

27. Park JH, Koh KC, Choi MS, et al. Analysis of risk factors associated with early multinodular recurrences after hepatic resection for hepatocellular carcinoma. Am J Surg 2006;192:29-33. 
28. Kim BK, Shim JH, Kim SU, et al. Risk prediction for patients with hepatocellular carcinoma undergoing chemoembolization: development of a prediction model. Liver Int 2016;36:92-99.

29. Adhoute X, Penaranda G, Naude S, et al. Retreatment with TACE: the ABCR SCORE, an aid to the decision-making process. J
Hepatol 2015;62:855-862.

30. Sieghart W, Hucke F, Pinter M, et al. The ART of decision making: retreatment with transarterial chemoembolization in patients with hepatocellular carcinoma. Hepatology 2013;57:2261-2273. 